\title{
Raised serum uric acid is associated with higher bone mineral density in a cross-sectional study of a healthy Indian population
}

This article was published in the following Dove Press journal:

Therapeutics and Clinical Risk Management

\section{Neelam Kaushal' \\ Divya Vohora' \\ Rajinder K Jalali² \\ Sujeet $\mathrm{Jha}^{3}$}

'Pharmaceutical Medicine, Department of Pharmacology, School of Pharmaceutical Education and Research, Jamia Hamdard, New Delhi, ${ }^{2}$ Medical Affairs and Clinical Research, Sun Pharmaceutical Industries Limited, Gurgaon, ${ }^{3}$ Institute of Endocrinology, Diabetes and Metabolism, Max Healthcare Inst. Ltd, New Delhi, India
Correspondence: Divya Vohora

Pharmaceutical Medicine, School of Pharmaceutical Education and Research, Jamia Hamdard, Mehrauli-Badarpur Road, Near Batra Hospital, Hamdard Nagar, New Delhi I 10062, India

Tel +9 I I I 26059688

Email divyavohora@hotmail.com

Sujeet Jha

Institute of Endocrinology, Diabetes and Metabolism, Max Healthcare Inst. Ltd., Press Enclave Road, Saket, New Delhi II0017, India

Email sujeet.jha@maxhealthcare.com
Purpose: Oxidative stress has been implicated as a fundamental mechanism in the decline of bone mass. Reactive oxygen species are reported to suppress osteoblast generation and differentiation and enhance osteoclast development and activity. Increasing evidence suggests favorable effect of serum uric acid (UA) on bone metabolism due to its antioxidant properties. Therefore, we investigated the association between serum UA levels and bone mineral density (BMD) in healthy adult Indian subjects.

Materials and methods: We reviewed the medical records of 524 subjects who had undergone preventive health check-ups in a tertiary care hospital that included UA and BMD measurements at femur neck, total femur, and lumbar spine. Subjects concomitantly taking drugs or having a medical condition that would affect the bone metabolism or UA levels were excluded.

Results: The final analysis included 310 subjects (mean age: $47.2 \pm 12.2$ years; females: $43.5 \%$; males: $56.5 \%$ ). Study population was categorized into two groups based on the group median value for UA (ie, $5.4 \mathrm{mg} / \mathrm{dL}$ ). BMD was significantly higher at all skeletal sites in subjects with UA $>5.4 \mathrm{mg} / \mathrm{dL}$ compared to subjects with $\mathrm{UA} \leq 5.4 \mathrm{mg} / \mathrm{dL}(p<0.001)$. On correlation analysis, UA was positively associated with BMD at all skeletal sites $(r=0.211-0.277 ; p<0.05)$. The correlation remained significant after controlling for age $(p<0.05)$ and lifestyle factors (smoking, alcohol use, physical activity, and diet; $p<0.05$ ) independently. UA significantly $(p<0.001)$ accounted for $4.5 \%-7.7 \%$ of the variance in BMD $\left(r^{2}=0.045-0.077\right)$ in unadjusted model and $1.6 \%-3.2 \%$ of the variance $(p<0.05)$ when adjusted for age and body mass index combined at lumbar spine and right femur neck, respectively.

Conclusion: We conclude that raised UA levels are associated with higher BMD at all skeletal sites and UA may have a protective role in bone metabolism owing to its antioxidant effect.

Keywords: oxidative stress, antioxidant effect, bone mineral density, uric acid, osteoporosis, T-scores

\section{Introduction}

Osteoporosis is a disease characterized by reduction in the bone mass and disruption of bone architecture leading to impaired skeletal strength and an increased susceptibility to fractures. ${ }^{1}$ It is estimated that over 200 million people worldwide have osteoporosis and the prevalence is continuing to escalate. The major complication of osteoporosis is an increase in fragility fractures leading to morbidity, mortality, and decreased quality of life. ${ }^{2}$ The number of osteoporosis patients in India was 26 million in 2003; while in 2013, 50 million people in India were either osteoporotic or had low bone mass. A study showed an annual incidence rate for hip fractures as 163 and 121 per 100,000 per year in women and men, respectively, above the age of 55 years. ${ }^{3}$ 
Recent evidence indicates oxidative stress as a fundamental mechanism of the age-dependent decline of bone mass and strength. ${ }^{4,5}$ As in other age-related diseases, there is an increased risk of oxidative stress, excessive production of reactive oxygen species, and decreased effectiveness of the antioxidant defense system in osteoporosis too. ${ }^{6-8}$ Reactive oxygen species are reported to suppress osteoblast generation and differentiation as well as enhance osteoclast development and activity. ${ }^{9,10}$ Many studies have shown that lower levels of plasma antioxidants such as plasma vitamins $\mathrm{C}$, $\mathrm{E}$, and A, plasma glutathione peroxidase, and $\alpha$-lipoic acid may be associated with a higher risk of osteoporosis and bone loss. ${ }^{11,12}$

Uric acid (UA) is a final enzymatic product in the degradation of purine nucleosides and free bases in humans. ${ }^{13}$ Increased UA is considered a risk factor for various chronic diseases, such as hypertension, diabetes mellitus, and cardiovascular diseases. ${ }^{14-16}$ However, contrary to this, there is an increasing evidence that UA has an important role as an antioxidant. ${ }^{17,18}$ Studies have shown that UA may have an advantageous effect in cancer ${ }^{19}$ and nervous system diseases such as multiple sclerosis, amyotrophic lateral sclerosis, Alzheimer's disease, and dementia. ${ }^{20-23}$ Similarly, it is hypothesized that serum UA may potentially protect against metabolic bone diseases, such as osteoporosis, owing to its antioxidant effects. Many studies across the globe have evaluated the relationship between UA and bone health, with most of the cross-sectional studies supporting the positive effect of UA on bone health. To the best of our knowledge, there is scanty data evaluating the relationship between UA and the bone mineral density (BMD) in Indian population. This study investigated the same in a cross-sectional study in India.

\section{Materials and methods Study design}

In this single-center, cross-sectional study, retrospective data were collected in Max Super Speciality Hospital, Saket, New Delhi (a tertiary care hospital). Data collection was initiated only after requisite approvals were obtained from the Scientific Committee and the Institutional Ethics Committee of Max Super Speciality Hospital. The requirement for informed consent was waived in view of the retrospective nature of the study and there being no direct contact with the study subjects. This study did not involve any intervention or therapy, and the research involved no risks to the subjects. Subjects' names and identity were not disclosed in any way during or after this database review study. Subjects were identified by subject ID numbers only, and hence, patient data confidentiality has been maintained.

\section{Subject screening, inclusion and exclusion criteria}

The medical records of adult male and female subjects who had voluntarily visited the hospital for general health check-ups were reviewed. These subjects had willingly chosen the health plans including measurement of BMD and serum UA. The data were screened based on prespecified inclusion and exclusion criteria as detailed ahead. Subjects with any medical condition which would influence bone metabolism, such as diabetes, cancer, hyperlipidemia, and hyperthyroidism, were excluded from the study. Subjects concomitantly taking drugs affecting the bone metabolism, such as bisphosphonates, steroids, anticoagulants (heparin), anticonvulsants, barbiturates, chemotherapeutic agents, gonadotropin-releasing hormone agonists, immunosuppressants, lithium, and long-acting progestin, were also excluded. Subjects on drugs affecting UA levels, such as allopurinol, probenecid, colchicine, diuretics, immunosuppressive agents, and salicylates, were not included. Subjects with gout were excluded too.

\section{Data collection}

Gender, age (years), weight $(\mathrm{kg})$ and height $(\mathrm{m})$, and body mass index (BMI; $\mathrm{kg} / \mathrm{m}^{2}$ ) were recorded. History of smoking, alcohol consumption, and exercise status were recorded for presence/absence, respectively; and dietary habits were recorded as vegetarian/nonvegetarian diet. Medication history and history of medical disorder were recorded. During health check-ups, subjects had undergone bone scanning with dual-energy-X-ray absorptiometry machine. The absolute areal BMD values $\left(\mathrm{g} / \mathrm{cm}^{2}\right)$ and $\mathrm{T}$-scores were available for five bone sites, that is, lumbar spine (L1-L4), femoral neck (both right and left), and total femur (both right and left). Laboratory data were collected for UA, total cholesterol, low-density lipoprotein, very low density lipoprotein, high-density lipoprotein, triglycerides, alkaline phosphatase, serum calcium, serum phosphate, fasting glucose (all measured in $\mathrm{mg} / \mathrm{dL}$ ), glycosylated hemoglobin (\%), and vitamin D (ng/mL).

\section{Statistical analysis}

Statistical analysis was performed using SPSS-20.0 for the Windows Statistical Package (IBM Corporation, Armonk, NY, USA). Descriptive data were presented as mean \pm SD or number (\%), unless specified. Univariate analysis was 
done by Student's $t$-test and chi-square test as appropriate. Pearson's correlation analysis was done to assess the relationship between BMD at various skeletal sites and UA. We reassessed the relationship by partial correlation analysis after adjustment for the possible confounders such as age, BMI, smoking, alcohol use, physical activity, and diet type. Multiple regression analysis was performed to investigate the strength of association of UA with bone site specific BMD after adjustment for age and BMI. A two-sided $p$-value $<0.05$ was considered statistically significant.

\section{Results}

\section{Subject characteristics}

The medical records of 524 subjects were reviewed, and the final analysis included 310 subjects (mean age of $47.2 \pm 12.2$ years [range: $20-84$ years]) after applying the inclusion and exclusion criteria detailed above. The study population consisted of 135 females $(43.5 \%)$ and 175 males $(56.5 \%)$. Based on the median value of UA $(5.4 \mathrm{mg} / \mathrm{dL})$ for the population, it was stratified into two groups: low UA group (UA levels $\leq 5.4 \mathrm{mg} / \mathrm{dL} ; \mathrm{n}=156$ ) and high UA group (UA levels $>5.4 \mathrm{mg} / \mathrm{dL} ; \mathrm{n}=154$ ). The baseline characteristics and laboratory parameters of the study population stratified by median UA levels are depicted in Table 1.

Height, weight, and BMI were significantly higher in high UA group $(p<0.001)$. Subjects in high UA group had significantly higher levels of low-density lipoprotein, triglycerides, and calcium, as compared to those in low UA group $(p<0.05)$. Significantly, low levels of high-density lipoprotein were found in high UA group as compared to low UA group $(p<0.001)$. There were no differences between the two groups with regards to alkaline phosphatase, serum phosphate and vitamin D, glucose (fasting), and glycosylated hemoglobin $(p>0.05$; Table 1$)$.

Table I Baseline characteristics and laboratory parameters of the study population stratified by uric acid status

\begin{tabular}{|c|c|c|c|c|}
\hline & $\begin{array}{l}\text { All subjects } \\
(\mathrm{N}=3 \mid 0)\end{array}$ & $\begin{array}{l}\text { Low uric acid group } \\
(n=\mid 56)\end{array}$ & $\begin{array}{l}\text { High uric acid group } \\
(n=154)\end{array}$ & $p$-value \\
\hline \multicolumn{5}{|l|}{ Demographic characteristics } \\
\hline Age (years) & $47.2 \pm 12.2$ & $48.0 \pm 12.3$ & $46.4 \pm 12.1$ & 0.261 \\
\hline Height (m) & $1.7 \pm 0.1$ & $1.6 \pm 0.1$ & $1.7 \pm 0.1$ & $<0.00 I^{*}$ \\
\hline Weight (kg) & $73.9 \pm 13.5$ & $68.1 \pm 11.3$ & $79.7 \pm 13.0$ & $<0.001 *$ \\
\hline BMI $\left(\mathrm{kg} / \mathrm{m}^{2}\right)$ & $26.7 \pm 3.9$ & $25.7 \pm 3.4$ & $27.7 \pm 4.1$ & $<0.00 I^{*}$ \\
\hline Female (\%) & 43.5 & 33.9 & 9.7 & $<0.00 I^{*}$ \\
\hline Male (\%) & 56.5 & 16.5 & 40.0 & \\
\hline \multicolumn{5}{|l|}{ Lifestyle characteristics $^{\mathrm{a}}$} \\
\hline Nonsmoker (\%) & 85.4 & 45.5 & 39.9 & $0.014^{*}$ \\
\hline Smoker (\%) & 14.6 & 4.9 & 9.7 & \\
\hline No alcohol consumption (\%) & 71.4 & 40.6 & 30.8 & $0.001 *$ \\
\hline Alcohol consumption present (\%) & 28.6 & 9.7 & 18.8 & \\
\hline No exercise (\%) & 5.5 & 2.9 & 2.6 & 0.824 \\
\hline Exercise present (\%) & 94.5 & 47.4 & 47.1 & \\
\hline Vegetarian diet (\%) & 39.1 & 20.4 & 18.8 & 0.557 \\
\hline Nonvegetarian diet (\%) & 60.9 & 29.6 & 31.2 & \\
\hline \multicolumn{5}{|l|}{ Laboratory parameters } \\
\hline Alkaline phosphatase (mg/dL) & $68.2 \pm 21.0$ & $67.3 \pm 22.7$ & $69.1 \pm 19.1$ & 0.470 \\
\hline Calcium (mg/dL) & $9.3 \pm 0.4$ & $9.2 \pm 0.3$ & $9.3 \pm 0.4$ & $0.017 *$ \\
\hline Phosphorus (mg/dL) & $3.5 \pm 0.5$ & $3.5 \pm 0.5$ & $3.5 \pm 0.5$ & 0.503 \\
\hline Glucose (fasting) (mg/dL) & $101.5 \pm 25.5$ & $101.6 \pm 28.4$ & $101.3 \pm 22.4$ & 0.910 \\
\hline Glycosylated hemoglobin (\%) & $5.6 \pm 0.8$ & $5.6 \pm 0.9$ & $5.7 \pm 0.7$ & 0.788 \\
\hline Total cholesterol (mg/dL) & $183.6 \pm 38.3$ & $179.7 \pm 39.0$ & $187.6 \pm 37.3$ & 0.071 \\
\hline $\mathrm{HDL}(\mathrm{mg} / \mathrm{dL})$ & $42.3 \pm 9.7$ & $45.6 \pm 10.7$ & $39.0 \pm 7.2$ & $<0.00 I^{*}$ \\
\hline LDL (mg/dL) & $120.7 \pm 34.9$ & $113.7 \pm 35.9$ & $127.8 \pm 32.5$ & $<0.00 I^{*}$ \\
\hline VLDL (mg/dL) & $29.6 \pm 21.4$ & $27.7 \pm 26.4$ & $31.5 \pm 14.5$ & 0.124 \\
\hline Triglycerides (mg/dL) & $144.2 \pm 84.2$ & $|3| . \mid \pm 93.0$ & $157.3 \pm 72.4$ & $0.006 *$ \\
\hline Uric acid (mg/dL) & $5.4 \pm 1.3$ & $4.3 \pm 0.8$ & $6.5 \pm 0.7$ & $0.001 *$ \\
\hline 25 hydroxyvitamin $D(\mathrm{ng} / \mathrm{dL})$ & $21.1 \pm 16.7$ & $19.3 \pm 12.6^{b}$ & $22.8 \pm 19.8^{c}$ & 0.285 \\
\hline
\end{tabular}

Notes: All absolute values are presented as mean \pm SD. ${ }^{a}$ Percentages for "total" are shown. Low uric acid group: uric acid $\leq 5.4$ mg/dL; high uric acid group: uric acid $>5.4 \mathrm{mg} / \mathrm{dL}$. ${ }^{b} \mathrm{n}=50,{ }^{c} \mathrm{n}=53$. ${ }^{*} p$-value $<0.05$.

Abbreviations: BMI, body mass index; HDL, high-density lipoprotein; LDL, low-density lipoprotein; VLDL, very low-density lipoprotein. 


\section{Comparative BMD and T-scores between the two UA groups}

BMD at all bone sites was significantly higher in subjects with higher UA levels $(p<0.001)$ as compared to subjects in low UA group (Table 2). Subjects with higher UA levels had significantly higher T-scores at lumbar spine, left femur neck, and right femur neck $(p<0.05)$; however, T-scores at left total femur $(p=0.137)$ and right total femur $(p=0.188)$ were comparable between the two UA groups. The prevalence of osteoporosis (T score $\leq-2.5$ ) was numerically higher at all bone sites in the low UA group; it was significantly higher at left total femur only ( $p=0.025$; Table 2$)$.

\section{Pearson's correlation analysis between $\mathrm{UA}$ and BMD}

UA was found to be positively and significantly associated with BMD at all bone sites ( $p<0.05$; Table 3 ). The Pearson's correlation coefficients $(r)$ were $0.211,0.263,0.277,0.275$, and 0.273 at lumbar spine, left femur neck, right femur neck, left total femur, and right total femur, respectively. The correlation of UA with BMD remained significant at all bone sites after independently controlling for age $(p<0.05)$ and lifestyle factors (smoking, alcohol use, physical activity,

Table 2 Absolute BMD and T-scores stratified by uric acid status

\begin{tabular}{|c|c|c|c|c|}
\hline & $\begin{array}{l}\text { All subjects } \\
(\mathrm{N}=3 \mid 0)\end{array}$ & $\begin{array}{l}\text { Low uric } \\
\text { acid group } \\
(n=156)\end{array}$ & $\begin{array}{l}\text { High uric } \\
\text { acid group } \\
(n=154)\end{array}$ & $p$-value \\
\hline \multicolumn{5}{|l|}{$\operatorname{BMD}\left(\mathrm{g} / \mathrm{cm}^{2}\right)$} \\
\hline Lumbar spine & I. $129 \pm 0.157$ & $1.097 \pm 0.152$ & $1.161 \pm 0.156$ & $0.001 *$ \\
\hline Left femur neck & $0.959 \pm 0.145$ & $0.926 \pm 0.133$ & $0.992 \pm 0.150$ & $0.001 *$ \\
\hline Right femur neck & $0.960 \pm 0.136$ & $0.926 \pm 0.127$ & $0.995 \pm 0.137$ & $0.001 *$ \\
\hline Left total femur & $0.987 \pm 0.137$ & $0.956 \pm 0.136$ & $1.019 \pm 0.132$ & $0.00 I^{*}$ \\
\hline Right total femur & $0.982 \pm 0.131$ & $0.953 \pm 0.132$ & $1.012 \pm 0.124$ & $0.001 *$ \\
\hline \multicolumn{5}{|c|}{ T-scores } \\
\hline Lumbar spine & $-0.5 \pm 1.2$ & $-0.7 \pm 1.2$ & $-0.4 \pm 1.2$ & $0.013^{*}$ \\
\hline Left femur neck & $-0.7 \pm 1.0$ & $-0.8 \pm 0.90$ & $-0.5 \pm 1.1$ & $0.003 *$ \\
\hline Right femur neck & $-0.6 \pm 0.96$ & $-0.8 \pm 0.80$ & $-0.5 \pm 1.0$ & $0.003 *$ \\
\hline Left total femur & $-0.5 \pm 0.97$ & $-0.6 \pm 1.0$ & $-0.4 \pm 0.90$ & 0.137 \\
\hline Right total femur & $-0.5 \pm 0.93$ & $-0.6 \pm 0.90$ & $-0.4 \pm 0.80$ & 0.188 \\
\hline \multicolumn{5}{|c|}{ Subjects, n (\%) with T-scores $\leq-2.5$ (osteoporosis) $^{\mathrm{a}}$} \\
\hline Lumbar spine & $20(6.5)$ & $14(4.5)$ & $6(1.9)$ & 0.069 \\
\hline Left femur neck & $14(4.5)$ & $10(3.2)$ & $4(1.3)$ & 0.106 \\
\hline Right femur neck & $9(2.9)$ & $6(1.9)$ & $3(1.0)$ & 0.320 \\
\hline Left total femur & $5(1.6)$ & $5(1.6)$ & 0 & $0.025 *$ \\
\hline Right total femur & $7(2.3)$ & $6(1.9)$ & I (0.3) & 0.058 \\
\hline
\end{tabular}

Notes: All absolute values are presented as mean \pm SD. Percentages for "total" are shown. Low uric acid group: uric acid $\leq 5.4 \mathrm{mg} / \mathrm{dL}$; high uric acid group: uric

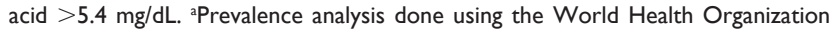
classification based on T-score: normal BMD (T-score $\geq-1$ ), osteopenia (T-score $<-I$ and $>-2.5$ ), and osteoporosis (T-score $\leq-2.5$ ). ${ }^{*} p$-value $<0.05$. Abbreviation: BMD, bone mineral density.
Table 3 Pearson's correlation coefficient ( $r$ ) between uric acid concentration and BMD

\begin{tabular}{llllll}
\hline Model & \multicolumn{3}{l}{$\begin{array}{l}\text { Lumbar } \\
\text { spine }\end{array}$} & neft femur & \multicolumn{3}{l}{$\begin{array}{l}\text { Right femur } \\
\text { neck }\end{array}$} & \multicolumn{2}{l}{$\begin{array}{l}\text { Left total } \\
\text { femur }\end{array}$} & $\begin{array}{l}\text { Right total } \\
\text { femur }\end{array}$ \\
\hline $\mathrm{I}$ & $0.211^{*}$ & $0.263^{*}$ & $0.277^{*}$ & $0.275^{*}$ & $0.273^{*}$ \\
2 & $0.198^{*}$ & $0.250^{*}$ & $0.265^{*}$ & $0.264^{*}$ & $0.263^{*}$ \\
3 & $0.136^{*}$ & $0.182^{*}$ & $0.194^{*}$ & $0.180^{*}$ & $0.182^{*}$ \\
4 & $0.167^{*}$ & $0.216^{*}$ & $0.242^{*}$ & $0.237^{*}$ & $0.240^{*}$ \\
5 & $0.179^{\$}$ & $0.258^{*}$ & $0.312^{*}$ & $0.318^{*}$ & $0.321^{*}$ \\
\hline
\end{tabular}

Notes: I, Uncontrolled bivariate correlation; 2, age-controlled partial correlation; 3, age- and BMI-controlled partial correlation; 4, smoking-, alcohol use-, physical activity-, and diet-controlled partial correlation; 5, 25-hydroxyvitamin D-controlled partial correlation. ${ }^{*} p<0.05,{ }^{\$} p=0.072$.

Abbreviations: BMD, bone mineral density; BMI, body mass index.

and diet adjusted; $p<0.05$ ) in partial correlation analysis. When controlled for age and BMI combined, the correlation remained significant at all bone sites $(p<0.05)$. The correlation remained significant even after controlling for hydroxyvitamin D at all skeletal sites except lumbar spine $(p=0.072)$.

\section{Linear regression analysis}

In simple linear regression analysis, significant $R$ square $\left(r^{2}\right)$ values $(p<0.001)$ were obtained at all skeletal sites. UA significantly $(p<0.05)$ contributed toward BMD variability at all bone sites $(4.5 \%, 6.9 \%, 7.7 \%, 7.6 \%$, and $7.5 \%$ variability at lumbar spine, left femur neck, right femur neck, total femur left, and right total femur, respectively). Regression "standardized coefficients ( $\beta$ )" for UA were positive in value and ranged from 0.211 to 0.277 for unadjusted model (Table 4). UA showed highest contribution of $7.7 \%$ toward BMD variance at right femur neck.

In age- and BMI-adjusted regression analysis (Table 5), the contribution of UA toward BMD remained significant $(p<0.05)$ at each skeletal site $(1.6 \%, 2.7 \%, 3.2 \%, 2.8 \%$, and $2.8 \%$ variability at lumbar spine, left femur neck, right femur neck, left total femur, and right total femur, respectively). In age- and BMI-adjusted analysis, standardized coefficient $(\beta)$ values were $0.133,0.174,0.187,0.174$, and 0.176 at lumbar spine, left femur neck, right femur neck, left total femur, and right total femur, respectively (not presented in Table 5).

\section{Discussion}

We investigated the correlation of BMD and UA in 310 adult Indian subjects having no such significant medical or medication history which could have affected BMD or UA. Our study results indicate that UA has a positive and significant association with BMD at all skeletal sites, which 
Table 4 Simple linear regression between uric acid and BMD

\begin{tabular}{lllllll}
\hline & $R$ & $\boldsymbol{R}^{2}$ & Adjusted $\boldsymbol{R}^{2}$ & $\begin{array}{l}\text { Unstandardized } \\
\text { coefficients }\end{array}$ & $\begin{array}{l}\text { Standardized } \\
\text { coefficients }(\boldsymbol{\beta})\end{array}$ \\
\hline Lumbar spine & $0.21 \mathrm{I}$ & 0.045 & $0.04 \mathrm{I}$ & 0.025 & $0.21 \mathrm{l}$ & 0.00 I $^{*}$ \\
Left femur neck & 0.263 & 0.069 & 0.066 & 0.029 & 0.263 & $0.00 I^{*}$ \\
Right femur neck & 0.277 & 0.077 & 0.074 & 0.029 & 0.277 & $0.00 I^{*}$ \\
Left total femur & 0.275 & 0.076 & 0.073 & 0.029 & 0.275 & $0.00 I^{*}$ \\
Right total femur & 0.273 & 0.075 & 0.072 & 0.027 & 0.273 & $0.00 I^{*}$ \\
\hline
\end{tabular}

Notes: Dependent variable: BMD; independent variable: uric acid. ${ }^{*} p<0.05$.

Abbreviation: BMD, bone mineral density.

is in concurrence with most of the earlier reported crosssectional studies. The association has been evaluated across the globe: in Australia, $,{ }^{24,25} \mathrm{China},{ }^{26,27} \mathrm{Japan},{ }^{28} \mathrm{Korea},{ }^{29-31}$ Thailand, ${ }^{32}$ the Netherlands, ${ }^{33}$ Turkey, ${ }^{34}$ Italy, ${ }^{35}$ and USA. ${ }^{36-38}$ The findings of our analysis are consistent with the literature which supports the hypothesis of UA being favorable to bone metabolism in humans.

For instance, in a cross-sectional study in men aged $\geq 70$ years $(n=1,705)$ in Australia, BMD at all sites was significantly higher among men with serum UA above the group median of $0.36 \mathrm{mmol} / \mathrm{L}(6.4 \mathrm{mg} / \mathrm{dL})$ than among men with UA below the median. Serum UA was associated with BMD at all sites $(\beta=0.12-0.14, p<0.001)$ and accounted for $1.0 \%-1.44 \%$ of the variances in BMD. ${ }^{24}$ Similarly, cross-sectional data analyses from Australian women $(n=356)$, mean age 60.5 years, revealed that women with higher UA levels had significantly higher absolute BMD at all skeletal sites. On longitudinal analyses, significant associations between serum UA levels and annual rates of change in BMD were seen at all skeletal sites. ${ }^{25}$ Likewise, UA was significantly and positively associated with the BMD after adjustment for multiple covariates in Chinese adults aged $40-75$ years $(n=3,079)$ in another cross-sectional analysis. ${ }^{26} \mathrm{~A}$ high serum UA level was associated with a high BMD, T-score, and $z$ score, and with low odds ratios for at least osteopenia and osteoporosis in males aged $\geq 50$ years and in postmenopausal females in another retrospective cross-sectional study $(\mathrm{n}=17,735)$ in China. ${ }^{27}$ A retrospective analysis of medical records of Japanese women (45-75 years, $\mathrm{n}=615$ ) concluded that higher UA levels were linearly associated with higher lumbar spine BMD. ${ }^{28}$ In a large cross-sectional study, in healthy Korean postmenopausal women ( $\mathrm{n}=7,502)$, after adjusting for multiple confounders, serum UA levels were positively associated with BMD at all sites (all $p<0.001$ ). Compared with the participants in the highest UA quartile, the odds for osteoporosis were $40 \%$ higher in those in the lowest quartile. ${ }^{29}$ Similarly, in a retrospective longitudinal study, including healthy Korean women aged $\geq 40$ years $(n=443)$ with an average follow-up interval of 2 years, serum UA level had a positive association

Table 5 Linear regression- $r^{2}$ change by uric acid in age- and BMI-adjusted model

\begin{tabular}{|c|c|c|c|c|c|c|c|}
\hline Model & $R$ & $R^{2}$ & Adjusted $R^{2}$ & $\begin{array}{l}\text { SE of the } \\
\text { estimate }\end{array}$ & $R^{2}$ change & F change & $\begin{array}{l}P \text {-values for } \\
F \text { change }\end{array}$ \\
\hline \multicolumn{8}{|c|}{ Lumbar spine } \\
\hline 1 & 0.347 & 0.120 & 0.114 & - & - & - & - \\
\hline 2 & 0.369 & 0.136 & 0.128 & 0.147 & 0.016 & 5.630 & $0.018^{*}$ \\
\hline \multicolumn{8}{|c|}{ Left femur neck } \\
\hline 1 & 0.414 & 0.171 & 0.166 & - & - & - & - \\
\hline 2 & 0.446 & 0.199 & 0.191 & 0.131 & 0.027 & 10.381 & $0.001 *$ \\
\hline \multicolumn{8}{|c|}{ Right femur neck } \\
\hline 1 & 0.401 & 0.161 & 0.155 & - & - & - & - \\
\hline 2 & 0.439 & 0.192 & 0.184 & 0.124 & 0.032 & 11.925 & $0.00 I^{*}$ \\
\hline \multicolumn{8}{|c|}{ Left total femur } \\
\hline 1 & 0.387 & 0.150 & 0.144 & - & - & - & - \\
\hline 2 & 0.421 & 0.178 & 0.169 & 0.126 & 0.028 & 10.156 & $0.002^{*}$ \\
\hline \multicolumn{8}{|c|}{ Right total femur } \\
\hline I & 0.380 & 0.144 & 0.139 & - & - & - & - \\
\hline 2 & 0.416 & 0.173 & 0.164 & 0.120 & 0.028 & 10.315 & $0.00 I^{*}$ \\
\hline
\end{tabular}

Notes: I, Predictors: age and BMI; 2, predictors: age, BMI, and uric acid. Dependent variable: BMD. ${ }^{*} p<0.05$. Abbreviations: BMD, bone mineral density; BMI, body mass index; SE, standard error. 
with the annual percentage changes in BMD. ${ }^{31}$ In a study in 1,320 males and 485 females aged 25-54 years in Thailand, when adjusted for covariates including age, body weight, and serum creatinine in multiple linear regression models, there was a positive association between UA levels and BMD at the lumbar spine in males only $(p<0.05)$. The association between UA levels and BMD was found in females after controlling for age, body weight, and serum creatinine at the femoral neck, but in the opposite direction $(p<0.05) .{ }^{32}$ Likewise, data analyses in 5,074 participants ( $\geq 55$ years) of The Rotterdam Study (the Netherlands) showed that higher levels of serum UA were associated with higher BMD. ${ }^{33}$ In a cross-sectional study in Turkish elderly ( $>70$ years, $n=2,190)$, UA was one of the independent variables predicting the occurrence of osteoporosis. Higher serum UA levels were associated with a lower prevalence of osteoporosis. ${ }^{34}$ In a case-control cohort study $(n=1,680)$ in USA, total hip BMD was significantly higher in the group of men with high UA compared with lower UA and increased linearly across quartiles of UA after multivariate adjustment ( $p$ for trend $=0.002$ ). ${ }^{36}$ Similarly, higher UA levels were associated with higher BMD in unadjusted analyses in 6,759 National Health and Nutrition Examination Survey, USA participants ( $>30$ years), but these associations were no longer statistically significant after adjustment for potential confounders. ${ }^{37}$

Not only higher BMD, but higher UA levels were also reported to be associated with lowered risk of fractures. In Australian men, higher serum UA was associated with a lower prevalence of fractures. ${ }^{24}$ In a longitudinal study of Korean men aged $\geq 50$ years $(n=16,078)$, baseline elevation of UA was significantly associated with a lower risk of incident fractures at osteoporosis-related sites during an average follow-up period of 3 years. ${ }^{30}$ In another study, Korean postmenopausal women with vertebral fractures had lower serum UA levels, compared with those without vertebral fractures $(p=0.009) .{ }^{29}$ Similarly, a study from USA reported that higher serum UA levels were associated with a reduction in risk of incident non-spine fractures, but not hip fractures. ${ }^{36}$

Contrary to these reports, baseline UA was not associated with the onset of new osteoporotic fractures over a 4.4-year follow-up in 1,586 Italian adults ( $>65$ years), that is, did not decrease the risk of osteoporotic fractures on follow-up. However, participants with higher UA levels had significantly less osteoporosis prevalence at baseline. ${ }^{35}$ Further, a U-shaped relationship between serum urate levels and hip fractures was reported in men aged $\geq 65$ years $(n=1,963)$ in USA on follow-up of 10 years. Men in the lowest and the highest urate quartiles ( $<4.88$ and $\geq 6.88 \mathrm{mg} / \mathrm{dL}$, respectively) had a significantly higher rate of fractures in unadjusted analysis. However, upon multivariate adjustment, only the hazard ratio for hip fracture in the highest quartile versus the reference remained significant. High serum urate levels were not associated with hip fractures in 2,729 women. ${ }^{38}$

Hence, the results of aforementioned studies majorly demonstrated a positive relationship between UA and bone health in Asian, Australian, Turkish, and Netherlander populations in both cross-sectional and longitudinal analyses. Though cross-sectional data from Italy and USA favor the proposition, the longitudinal data are not supportive. The answer to contradictory results may be elucidated by different demographic characteristics such as gender, age, ethnicity, study designs, number of subjects, and confounders controlled for while analyzing the data. Literature supports the occurrence of ethnic differences in BMD, and genetic variation may be one of the potential explanations for the ethnic variation in BMD. ${ }^{39-42}$ The contributions of several gene polymorphisms regulating bone mass and its mineral content have been recognized, including polymorphisms of the $V D R, E S R, C T R$, and COL1A1 genes. Other candidate genes such as $A H S G$ and $P T H$ have also been investigated for their association with bone phenotypes by employing various polymorphic molecular markers. ${ }^{42,43}$ As reviewed by Lei et al, the osteoporosis-related phenotypic differences between Asians and Caucasians might be partially the result of the different ethnic genetic background. ${ }^{42}$

The hypothesis of positive relationship between UA and bone health has been reinforced by a recently published meta-analysis by Veronese et al of 19 studies including 55,859 participants. The authors analyzed data regarding $\mathrm{BMD}$, osteoporosis, and fractures in people with higher serum UA versus lower serum UA concentrations. Subjects with higher UA levels had significantly higher BMD values for the spine (six studies; standardized mean difference $[\mathrm{SMD}]=0.29 ; 95 \% \mathrm{CI}: 0.22-0.35 ; I^{2}=47 \%$ ), total hip (seven studies; SMD $=0.29 ; 95 \%$ CI: $0.24-0.34 ; I^{2}=33 \%$ ), and femoral neck (six studies; SMD $=0.25 ; 95 \%$ CI: 0.16-0.34; $I^{2}=71 \%$ ). Simple correlation analyses substantially confirmed these findings. An increase of $1 \mathrm{SD}$ in serum UA levels reduced the number of new fractures at follow-up (three studies; hazard ratio $=0.83 ; 95 \%$ CI: $0.74-0.92 ; I^{2}=0 \%$ ). No significant differences between men and women emerged, although data about women were limited. Hyperuricemia was found independently associated with BMD and fractures, supporting a protective role for UA in bone metabolism disorders. ${ }^{44}$ Furthermore, in another recently published study 
by Xiao et al, serum UA levels were positively correlated with lumbar spine BMD and T-values in Chinese Han males $(n=385)$ aged $>50$ years, suggesting the protective effect of UA on bone density at the lumbar spine. ${ }^{45}$

The exact mechanism of association between UA and BMD is unclear. The hypothesis is that UA in its crystalline state has inflammatory properties, whereas the soluble form within the normal physiologic levels may have antioxidant properties; $50 \%$ of the antioxidant properties of plasma are accounted for by UA. Due to the antioxidant properties, UA may inhibit osteoclastic bone resorption and contribute to higher BMD. ${ }^{36}$ An in vitro study showed that UA suppressed osteoclastogenesis in a dose-dependent manner and decreased the production of reactive oxygen species in osteoclast precursors, indicating UA may have beneficial effects on bone metabolism as an antioxidant. Investigators concluded that the mechanism appears to be through reduction in the number and activity of osteoclasts that resorb bone. ${ }^{29}$ Further, in another preclinical study, UA exerted arthroprotective effects against arthritis in oxonic acidtreated mice, as these mice displayed less inflammatory cell infiltration in the synovium, less synovial hyperplasia, less cartilage damage, and less bone erosion than control mice. ${ }^{46}$ However, more research is required to further characterize this observation.

\section{Limitations and strengths of the study}

Because this was a retrospective analysis, the study data are dependent on accurate and complete documentation in the medical records. The details of menopausal status were not available. Subjects in this study were not from general community, but from a single tertiary hospital, and subjects had come willingly for voluntary health check-ups. The study did not include longitudinal data. In view of the small sample size, relationship analysis could not be done for males and females separately.

Our study was a well-characterized cross-sectional study that excluded subjects with comorbid diseases or concomitant medication that would have influenced serum UA levels or BMD. To the best of our knowledge, this was the first Indian study evaluating the relationship between BMD and UA.

\section{Conclusion}

To conclude, raised serum UA levels were associated with higher BMD at all skeletal sites in this study, suggesting that UA may have a protective role in bone health possibly through its antioxidant action. However, the antioxidant theory is highly speculative and remains controversial till date. Therefore, more in-depth studies with a large sample size across geographies, as well as basic research are warranted to elucidate the role of UA as an antioxidant in bone metabolism.

\section{Acknowledgments}

We are thankful to Tertiary Care Hospital (Max Healthcare Inst. Ltd, Saket, New Delhi, India) for all the support provided in data collection for this study. We thank Laxmi Raghuvanshi, and Samreen Siddiqui, Max Healthcare Inst. Ltd, Saket, New Delhi, India, for their technical and data collection support.

\section{Author contributions}

All authors have contributed toward the conception, design of the study, data analysis, drafting and revising the paper, and agree to be accountable for all aspects of the work. Dr SJ has additionally been instrumental in acquisition of the data from the hospital.

\section{Disclosure}

The authors report no conflicts of interest in this work.

\section{References}

1. Lane NE. Epidemiology, etiology, and diagnosis of osteoporosis Am J Obstet Gynecol. 2006;194(2 Suppl):S3-S11.

2. Reginster JY, Burlet N. Osteoporosis: a still increasing prevalence. Bone. 2006;38(2 Suppl 1):S4-S9.

3. Mithal A, Bansal B, Kyer CS, et al. The Asia-Pacific Regional AuditEpidemiology, Costs, and Burden of Osteoporosis in India 2013: A report of International Osteoporosis Foundation. Indian J Endocrinol Metab. 2014;18(4):449-454.

4. Manolagas SC. From estrogen-centric to aging and oxidative stress: a revised perspective of the pathogenesis of osteoporosis. Endocr Rev 2010;31(3):266-300.

5. Karasik D, Cohen-Zinder M. The genetic pleiotropy of musculoskeletal aging. Front Physiol. 2012;3:303.

6. Mlakar SJ, Osredkar J, Prezelj J, et al. The antioxidant enzyme GPX1 gene polymorphisms are associated with low BMD and increased bone turnover markers. Dis Markers. 2010;29(2):71-80.

7. Sendur OF, Turan Y, Tastaban E, Serter M. Antioxidant status in patients with osteoporosis: a controlled study. Joint Bone Spine. 2009; 76(5):514-518.

8. Wilson C. Bone: Oxidative stress and osteoporosis. Nat Rev Endocrinol. 2014;10(1):3.

9. Kousteni S. FoxOs: Unifying links between oxidative stress and skeletal homeostasis. Curr Osteoporos Rep. 2011;9(2):60-66.

10. Meng SJ, Yu LJ. Oxidative stress, molecular inflammation and sarcopenia. Int J Mol Sci. 2010;11(4):1509-1526.

11. Mainini G, Rotondi M, Di Nola K, et al. Oral supplementation with antioxidant agents containing alpha lipoic acid: effects on postmenopausal bone mass. Clin Exp Obstet Gynecol. 2012;39(4):489-493.

12. Maggio D, Barabani M, Pierandrei M, et al. Marked decrease in plasma antioxidants in aged osteoporotic women: results of a cross-sectional study. J Clin Endocrinol Metab. 2003;88(4):1523-1527.

13. Sautin YY, Johnson RJ. Uric acid: the oxidant-antioxidant paradox. Nucleosides Nucleotides Nucleic Acids. 2008;27(6):608-619. 
14. Johnson RJ, Kang DH, Feig D, et al. Is there a pathogenetic role for uric acid in hypertension and cardiovascular and renal disease? Hypertension. 2003;41(6):1183-1190.

15. Dehghan A, van Hoek M, Sijbrands EJ, et al. High serum uric acid as a novel risk factor for type 2 diabetes. Diabetes Care. 2008;31(2): 361-362.

16. Verdecchia P, Schillaci G, Reboldi G, et al. Relation between serum uric acid and risk of cardiovascular disease in essential hypertension. The PIUMA study. Hypertension. 2000;36(6):1072-1078.

17. Glantzounis GK, Tsimoyiannis EC, Kappas AM, et al. Uric acid and oxidative stress. Curr Pharm Des. 2005;11(32):4145-4151.

18. Waring WS, Convery A, Mishra V, et al. Uric acid reduces exerciseinduced oxidative stress in healthy adults. Clin Sci (Lond). 2003;105(4): 425-430.

19. Taghizadeh N, Vonk JM, Boezen HM. Serum uric acid levels and cancer mortality risk among males in a large general population-based cohort study. Cancer Causes Control. 2014;25(8):1075-1080.

20. Massa J, O’Reilly E, Munger KL, et al. Serum uric acid and risk of multiple sclerosis. J Neurol. 2009;256(10):1643-1648.

21. Keizman D, Ish-Shalom M, Berliner S, et al. Low uric acid levels in serum of patients with ALS: further evidence for oxidative stress? J Neurol Sci. 2009;285(1-2):95-99.

22. Andreadou E, Nikolaou C, Gournaras F, et al. Serum uric acid levels in patients with Parkinson's disease: their relationship to treatment and disease duration. Clin Neurol Neurosurg. 2009;111(9):724-728.

23. Euser SM, Hofman A, Westendorp RG, et al. Serum uric acid and cognitive function and dementia. Brain. 2009;132(Pt 2):377-382.

24. Nabipour I, Sambrook PN, Blyth FM, et al. Serum uric acid is associated with bone health in older men: a cross-sectional population-based study. J Bone Miner Res. 2011;26(5):955-964.

25. Makovey J, Macara M, Chen JS, et al. Serum uric acid plays a protective role for bone loss in peri- and postmenopausal women: a longitudinal study. Bone. 2013;52(1):400-406.

26. Dong XW, Tian HY, He J, et al. Elevated Serum Uric Acid Is Associated with Greater Bone Mineral Density and Skeletal Muscle Mass in Middle-Aged and Older Adults. PLoS One. 2016;11(5):e01,54,692.

27. Lin X, Zhao C, Qin A, et al. Association between serum uric acid and bone health in general population: a large and multicentre study. Oncotarget. 2015;6(34):35395-35403.

28. Ishii S, Miyao M, Mizuno Y, et al. Association between serum uric acid and lumbar spine bone mineral density in peri- and postmenopausal Japanese women. Osteoporos Int. 2014;25(3):1099-1105.

29. Ahn SH, Lee SH, Kim BJ, et al. Higher serum uric acid is associated with higher bone mass, lower bone turnover, and lower prevalence of vertebral fracture in healthy postmenopausal women. Osteoporos Int. 2013;24(12):2961-2970.

30. Kim BJ, Baek S, Ahn SH, et al. Higher serum uric acid as a protective factor against incident osteoporotic fractures in Korean men: a longitudinal study using the National Claim Registry. Osteoporos Int. 2014;25(7):1837-1844.
31. Kim S, Jung J, Jung JH, et al. Risk Factors of Bone Mass Loss at the Lumbar Spine: A Longitudinal Study in Healthy Korean Pre- and Perimenopausal Women Older than 40 Years. PLoS One. 2015;10(8): e01,36,283.

32. Sritara C, Ongphiphadhanakul B, Chailurkit L, et al. Serum uric acid levels in relation to bone-related phenotypes in men and women. J Clin Densitom. 2013;16(3):336-340

33. Muka T, de Jonge EA, Kiefte-de Jong JC, et al. The Influence of Serum Uric Acid on Bone Mineral Density, Hip Geometry, and Fracture Risk: The Rotterdam Study. J Clin Endocrinol Metab. 2016; 101(3):1113-1122.

34. Kuyumcu ME, Yesil Y, Oztürk ZA, et al. The association between homocysteine (hcy) and serum natural antioxidants in elderly bone mineral densitometry (BMD). Arch Gerontol Geriatr. 2012;55(3):739-743.

35. Veronese N, Bolzetta F, De Rui M, et al. Serum uric acid and incident osteoporotic fractures in old people: The PRO.V.A study. Bone. 2015; 79:183-189.

36. Lane NE, Parimi N, Lui LY, et al; Osteoporotic Fractures in Men Study Group. Association of serum uric acid and incident nonspine fractures in elderly men: the Osteoporotic Fractures in Men (MrOS) study. J Bone Miner Res. 2014;29(7):1701-1707.

37. Zhang D, Bobulescu IA, Maalouf NM, et al. Relationship between serum uric acid and bone mineral density in the general population and in rats with experimental hyperuricemia. J Bone Miner Res. 2015; 30(6):992-999.

38. Mehta T, Bůžková P, Sarnak MJ, et al. Serum urate levels and the risk of hip fractures: data from the Cardiovascular Health Study. Metabolism. 2015;64(3):438-446.

39. Anderson JJ, Pollitzer WS. Ethnic and genetic differences in susceptibility to osteoporotic fractures. Adv Nutr Res. 1994;9:129-149.

40. Zengin A, Prentice A, Ward KA. Ethnic differences in bone health. Front Endocrinol (Lausanne). 2015;6:24.

41. Leslie WD. Clinical review: Ethnic differences in bone mass - clinical implications. J Clin Endocrinol Metab. 2012;97(12):4329-4340.

42. Lei SF, Chen Y, Xiong DH, et al. Ethnic difference in osteoporosisrelated phenotypes and its potential underlying genetic determination. J Musculoskelet Neuronal Interact. 2006;6(1):36-46.

43. Musumeci M, Vadalà G, Tringali G, et al. Genetic and environmental factors in human osteoporosis from Sub-Saharan to Mediterranean areas. J Bone Miner Metab. 2009;27(4):424-434.

44. Veronese N, Carraro S, Bano G, et al. Hyperuricemia protects against low bone mineral density, osteoporosis and fractures: a systematic review and meta-analysis. Eur J Clin Invest. 2016;46(11):920-930.

45. Xiao J, Chen W, Feng X, et al. Serum uric acid is associated with lumbar spine bone mineral density in healthy Chinese males older than 50 years. Clin Interv Aging. 2017;12:445-452.

46. Lai JH, Luo SF, Hung LF, et al. Physiological concentrations of soluble uric acid are chondroprotective and anti-inflammatory. Sci Rep. 2017;7(1):2359.
Therapeutics and Clinical Risk Management

\section{Publish your work in this journal}

Therapeutics and Clinical Risk Management is an international, peerreviewed journal of clinical therapeutics and risk management, focusing on concise rapid reporting of clinical studies in all therapeutic areas, outcomes, safety, and programs for the effective, safe, and sustained use of medicines. This journal is indexed on PubMed Central, CAS,

\section{Dovepress}

EMBase, Scopus and the Elsevier Bibliographic databases. The manuscript management system is completely online and includes a very quick and fair peer-review system, which is all easy to use. Visit http://www.dovepress.com/testimonials.php to read real quotes from published authors. 